\title{
Dissipated energy based fatigue crack propagation law for complex loading conditions
}

\author{
by O. Plekhov, A. Izumova, A. Vedernikova and A. Vshivkov
}

\begin{abstract}
Institute of Continuous Media Mechanics, Russian Academy of Sciences, Ak. Koroleva str 1, 614013 Perm,
\end{abstract}
Russia,poa@icmm.ru

\begin{abstract}
The experimental study of heat flux evolution at the fatigue crack tip during uniaxial and biaxial loading was carry out in this work. The plane samples of stainless steel AISI 304 and titanium alloy Grade 2 with were weakened by notch to initiate fatigue crack. During tests, the samples were subjected to cyclic loading with constant stress amplitude and different biaxial coefficient. Infrared thermography, lock-in thermography and the contact heat flux sensor based on the Seebeck effect are used to monitor the dissipated thermal energy. A linear correlation between the crack rate and dissipated energy was obtained by these techniques.
\end{abstract}

\section{Introduction}

During the last decades, the enhanced ability to detect temperature evolution in the crack tip based on infrared thermography, along with a great interest to the investigation of defect evolution in the fatigue crack tip. The heat generation process depends on both the thermo elastic effect and plastic energy dissipation. The theoretical and experimental investigation of this problem can be useful for explanation of many actual problems in fracture mechanics such as applicability of crack tip closure concept, energy storage in defect ensemble in process zone. The measurement of heat flow near the crack tip allows one to calculate the energy balance under crack propagation and to obtain a new equation for crack propagation.

One of the actual problems of the fracture mechanics is the forecast of lifetime of cracked metal parts of engineering constructions subjected to the cyclic loading. The process of fatigue crack propagation in metals is accompanied by intense strain localization in areas of monotonic and cyclic plastic deformation leads to the temperature perturbation at crack tip. Many authors investigated the correlation between crack propagation rate and intensity of energy storage and dissipation processes [1-20] and showed that the dissipated energy plays a key role in the crack growth. From experimental point of view, the dissipated energy is well-measured value and could be estimated by different experimental techniques.

The way for calculated of the power of the heat flow based on the analysis of evolution of the temperature field on the specimen surface by means IR thermography data. Heat sources field calculation is carried out with the using of heat conduction equation averaged on volume. This calculation has several problems. The measured temperature signals are noisy and heat exchange processes are ill-defined. It leads to low precision in the solution of inverse thermal conductivity problem. The problems was solved by combination of infrared measurement with contact heat flux sensor. This idea was affectively used for investigation of energy dissipation under liquid flow [3]. The infrared thermography allows us to study the spatial distribution of heat sources. The additional contact measurement allows us to measure the real value of heat flow and to calibrate the infrared measurements.

The IR data widely used to gather information for a better understanding of plastic deformation and fracture of metallic materials $[5-10]$. In works $[11,12]$ shows the satisfactory agreement of the results contact (heat flux sensor) and non-contact (IR thermography) measurements of dissipated energy during irreversible deformation. Proved the possibility using of these measurements in a combination (to verify the heat source value, its distribution over the material surface and heat exchange conditions for specimen and environment) or separately (as an express method to evaluate material conditions at different stages of loading).

Another technique is Lock-in thermography that allows space-resolved measurements and directly evaluate of elastic stress fields according to the thermo-elastic effect [13] and the investigation of dissipative energies with the double frequency method proposed by Sakagami [14]. The determination of dissipated energy is used to evaluate the crack initiation as well as effects in crack propagation experiments [15-20].

In this study, we have shown that the dissipated energy values estimated by Lock-In thermography technique and contact heat flux sensor have a good qualitative agreement.

Both techniques could be applied for quasi-static and cyclic loading. The study of quasi-static loading allows us to show that the stored energy rate peaks on the initial stage of plastic deformation and vanishes at developed plastic flow while the value of the stored energy reaches a certain critical value that means that materials prepared to the failure. The focus of this work is cyclic loading. The measured values dissipated energy under cyclic loading allows us to determine a linear correlation between the crack rate and dissipated energy (value of amplitude of second temperature harmonics) for predicting the fatigue crack growth. 


\section{Experimental setup}

A series of uniaxial and biaxial fatigue test were carry out on flat samples with stress concentrator made from stainless steel AISI 304 and titanium alloy Grade 2. The biaxial test was provided using servo-hydraulic biaxial testing system Biss BI-00-502, located in Kazan Scientific Center of Russian Academy of Sciences. The geometry of the samples is shown in figure 1. During tests the samples were subjected to cyclic loading with constant stress amplitude, different biaxial coefficient $\eta=P x / P y(1,0.5,0)$ and stress ratio $R=0.1$. The crack length was measured by an optic method. The uniaxial test was provided using servo-hydraulic testing machine Instron 8802 . The geometry of the samples is shown in figure 2. During crack propagation experiments the specimens were subjected to cyclic loading at a frequency $10 \mathrm{~Hz}$ with constant stress amplitude and $R=0.1$. For crack length measurement the DC potential drop method was used [21].

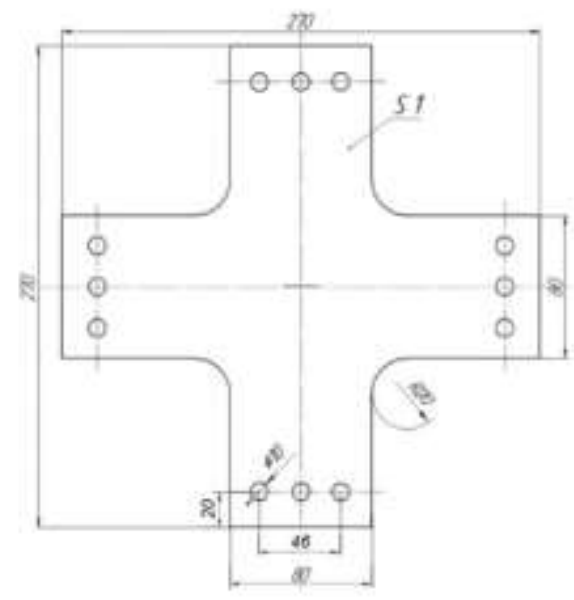

Fig. 1. Geometry of biaxial samples

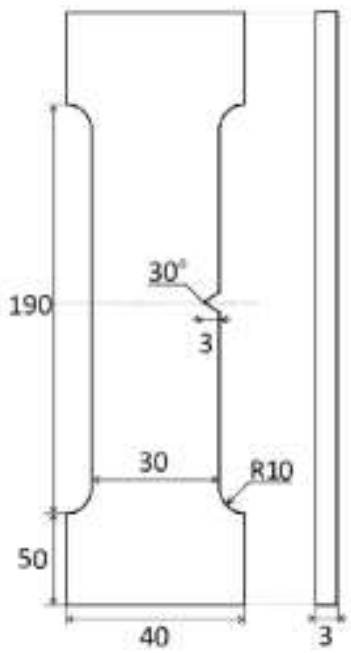

Fig. 2. Geometry of uniaxial samples

To analyse the heat flow power at the crack tip the Seebeck effect-based heat flux sensor developed in previous study was used [2]. To enhance the heat flow, a heat-conductive paste was applied between the sensor and the specimen.

The evolution of the temperature field was recorded in some time intervals by an IR camera FLIR SC 5000. IR camera has the following features: the spectral range of $3-5 \mu \mathrm{m}$, the maximum frame size is $320 \times 256$ pixels, the spatial resolution is 10-4 meters. The temperature sensitivity is in the range from $25 \mathrm{mK}$ to $300 \mathrm{~K}$. Calibration of the camera was made based on the standard calibration table. The application of the LIRSC5000 MW G1 F/3.0 close-up lens (with distortion less than $0.5 \%$ ) made it possible to investigate the plastic zone in detail. The specimen surface intended for infrared shooting was polished in several stages and coated by a thin layer of amorphous carbon to improve the surface emissivity. The thermography sequences with a length of $10 \mathrm{~s}$ at a frame rate $100 \mathrm{~Hz}$ were recorded and after analysed with the infrared imaging software suite Altair LI.

\subsection{Direct measurement of heat flux}

To analyse the energy balance at the crack tip a contact heat flux sensor was used [2]. The proposed sensor is based on the Seebeck effect, which is the reverse of the Peltier effect. The contact heat flux sensor is attached to the specimen during all mechanical test. To enhance the heat flow a heat-conductive paste was inserted between the sensor and the specimen. The quantity of heat absorbed or dissipated by the element is directly proportional to the current intensity and the time of its passage:

$$
P=\Pi_{A B} I
$$

where $P$ - the power of heat flow, $I$ - the direct current, $\Pi_{A B}$ - Peltier coefficient, which is related with a coefficient of thermal electromotive force.

\subsection{Lock-in thermography measurements}

The dissipated energy can be estimated by Lock-in thermography. Lock-in thermography is based on a correlation in frequency, amplitude and phase of the detected signal with a reference signal coming from the loading system. The evaluation is based on a Discrete Fourier Transformation (Eqn. 2) and performed for each pixel of the recorded frames. It was shown that in case of plastic deformation a second mode coupled with the double loading frequency appears (D- 
mode) and correlated with dissipative energies [14]. For each analysed sequence of frames, the evaluation provides an amplitude and a phase image for the different modes.

$$
T(t)=T_{m}+T_{E} \cdot \sin \left(2 \pi f_{L} \cdot t+\varphi_{E}\right)+T_{D} \cdot \sin \left(2 \cdot 2 \pi f_{L} \cdot t+\varphi_{D}\right)
$$

where $T_{m}$ - mean temperature, $f_{L}$ - mechanical loading frequency, $\varphi_{E}$ and $\varphi_{D}$ - phase shifts, $T_{E}$ - thermo-elastic amplitude (E-mode), $T_{D}$ - plasticity effects amplitude (D-mode).

Eq. (1) is integrated in the algorithm of Altair LI software, providing an image in data matrix form for each constant parameter. In figure 3 the resulting $D$-amplitude images of the lock-in evaluation of an experiment with a constant stress amplitude and a crack length of about $7 \mathrm{~mm}$ for both materials are shown. The D-amplitude image shows the plastic zone in front of the crack tip.

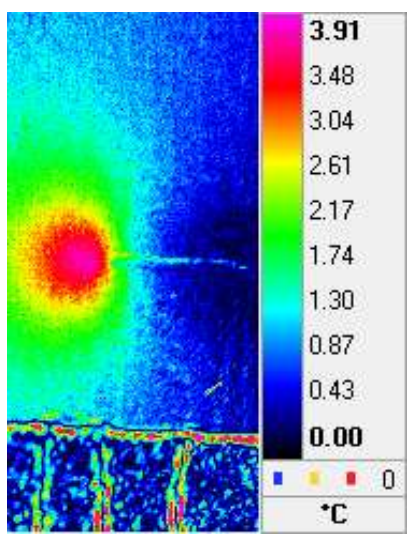

(a)

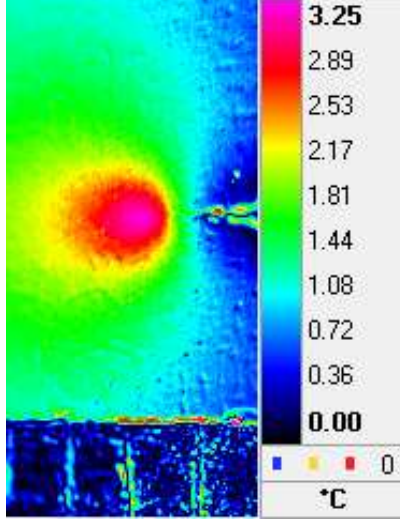

(b)

Fig. 3. D-Amplitude image of a specimens loaded with a constant stress amplitude for (a) Grade 2, (b) steel AISE 304.

\subsection{Estimation of the heat sources field based on the heat conductivity equation}

To calculate the heat source field induced by plastic deformation, we use heat conduction equation (3) for processing the obtained infrared thermography data:

$$
\rho c \frac{\partial T(x, y, z, t)}{\partial t}=Q(x, y, z, t)+k\left(\frac{\partial^{2} T(x, y, z, t)}{\partial x^{2}}+\frac{\partial^{2} T(x, y, z, t)}{\partial y^{2}}+\frac{\partial^{2} T(x, y, z, t)}{\partial z^{2}}\right)
$$

where $T(x, y, z, t)$ is the temperature field, $\rho$ is the material density $(\mathrm{kg} / \mathrm{m} 3), c$ is the heat capacity $(\mathrm{J} /(\mathrm{kg} \cdot \mathrm{K})), k$ is the heat conductivity $(\mathrm{W} /(\mathrm{m} \cdot \mathrm{K})), Q(x, y, z, t)$ is the heat source field, $x, y, z$ are the coordinates, and $t$ is the time.

The IR camera allows one to register the temperature distribution only over the specimen surface that is the reason why Eq. 2 has to be averaged over the z-coordinate (thickness).

Difference $\theta^{\prime}(x, y, t)$ between the averaged specimen temperature $T(x, y, z, t)$ and the initial specimen temperature in the thermal balance with the environment $T_{0}$ is defined as:

$$
\theta^{\prime}(x, y, t)=\frac{1}{h} \int_{-h / 2}^{h / 2}\left(T(x, y, z, t)-T_{0}\right) d z=\theta(x, y, t)-T_{0}
$$

where $b$ is the specimen thickness.

The following boundary conditions are considered:

$$
\left.\frac{\partial T(x, y, z, t)}{\partial x}\right|_{z=\frac{h}{2}}=-\left.\frac{\partial T(x, y, z, t)}{\partial z}\right|_{z=-\frac{h}{2}}-\left.k \frac{\partial T(x, y, z, t)}{\partial z}\right|_{z=\frac{h}{2}}=\frac{\beta}{h} \int_{-h / 2}^{h / 2}\left(T(x, y, ₹, t)-T_{0}\right) d z
$$


where $\beta$ is the heat exchange coefficient in perpendicular direction to the specimen surface. One boundary condition describes the symmetry of the heat source, whereas the second boundary condition is responsible for the heat exchange of the specimen with the environment.

Therefore, integrating Eq. (3), considering expressions (4) and boundary conditions (5), we obtain relation (6) to estimate the heat source field caused by irreversible deformation:

$$
Q_{\text {int }}(x, y, t)=\rho c\left(\dot{\theta}(x, y, t)+\frac{\theta(x, y, t)-T_{0}}{\tau}\right)-k \Delta \theta(x, y, t)
$$

where $\tau$ is the time constant, which is related to the heat losses [22, 23]. The parameter $\tau$ was measured before each test by the additional experimental procedure of specimen cooling after pulse point heating. The identification process consisted in estimating the time derivative and the Laplacian of the temperature function if there was no internal and external heat source on the specimen during its cooling. For steel AISI 304, the value of parameter $\tau$ amounted to 10 sec.

The numerical finite-difference scheme of equation (6) applied to the IR thermography data allows one to investigate the heat source evolution on the specimen surface. To calculate the heat sources from the noisy temperature fields, the procedure of the movement compensation and filtering of infrared data was performed. These algorithms are described in detail in [24].

\section{Results of experiments}

In order to compare the heat flux sensor results and the results of thermography measurements, we have studied the temperature evolution in a small rectangular area which covered all temperature fluctuations near the crack tip. The size of the area coincides with heat flux sensor dimensions.

A comparison of the results obtained by contact sensor, the infrared thermography data (heat conduction equation (1)) and lock-in thermography data during crack propagation tests is illustrated in figure 2 . The heat flux sensor allows measurement of the integral heat flux only. The infrared thermography technique was used to obtain the image of temperature distribution and the field of heat source distribution in the crack tip region. To compare the IR results with the data of the contact sensor, we integrated the heat source field over the space equal to the size of contact sensor.

With the Altair LI software it is possible to calculate the resulting amplitude of temperature variations (amplitude image) and the distribution of phase shifts between the thermographic signal and the mechanical loading (phase image) for the E-mode and D-mode, respectively (Eq. 7). As shown by Bremond [13], the D-mode provides information about the dissipated energy. The values of the amplitude related to the double loading frequency were determined.

Figure 4 presents the characteristic curve describing the heat flux variation during the fatigue tests obtained by heat flux sensor, infrared thermography technique and lock-in technique.

Three zones were identified on the heat flux curve during the crack propagation experiment. The short initial increasing zone corresponds to the crack initiation stage. The second zone with a constant heat flux corresponds to the steady state crack growth stage. The last zone is characterized by a sharp increase in heat dissipation and is ended with specimen failure. It can be seen that the power heat source detected by the contact sensor and determined on the basis of IR thermography data (equation (4)) are in good quantitative agreement throughout the test.

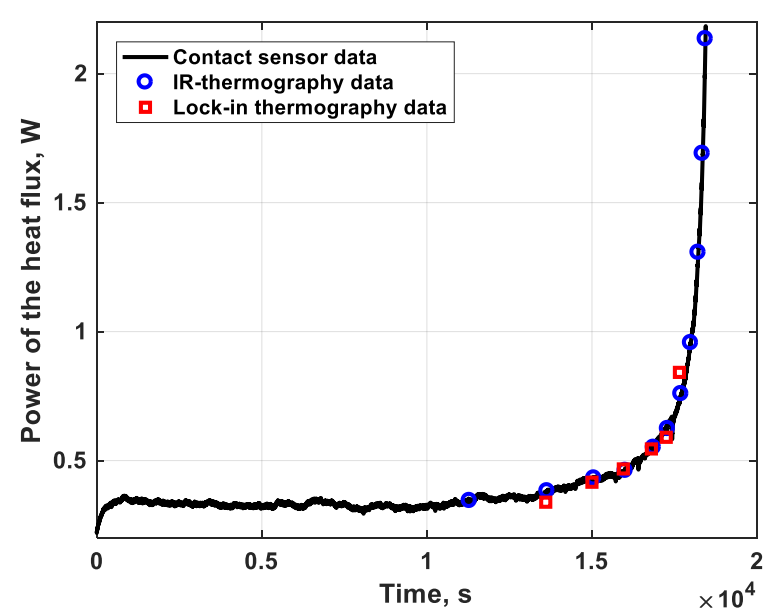

(a)

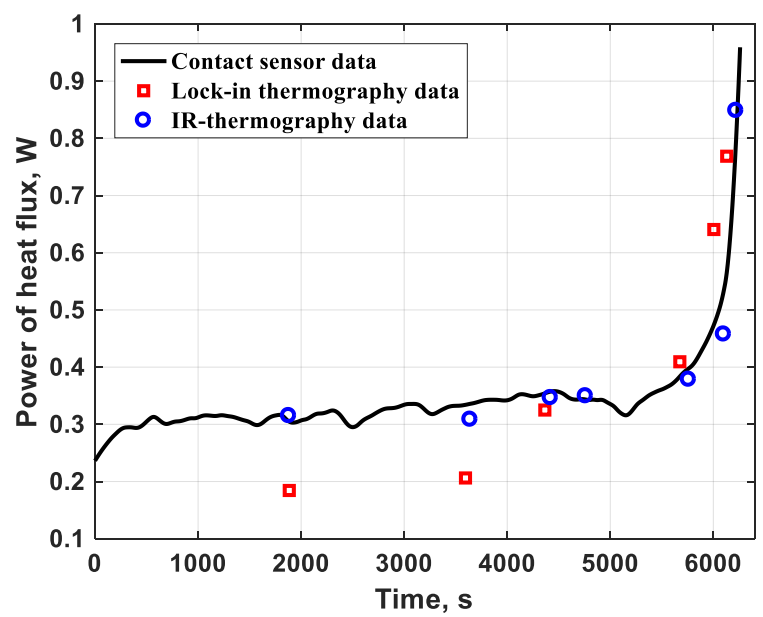

(b)

Fig. 4. Characteristic IR-thermography data, lock-in thermography data and heat flux sensor measurements for stainless steel AISI 304 (a) and titanium alloy Grade 2 (b). 
Figs. 5 present the relation between the heat flux power $Q$ and crack growth rate $d a / d N$ for the stainless steel and titanium alloy specimen. The power law relation for predicting the fatigue crack growth is determined as follows:

$$
d a / d N=a Q_{\text {int }}^{b}
$$

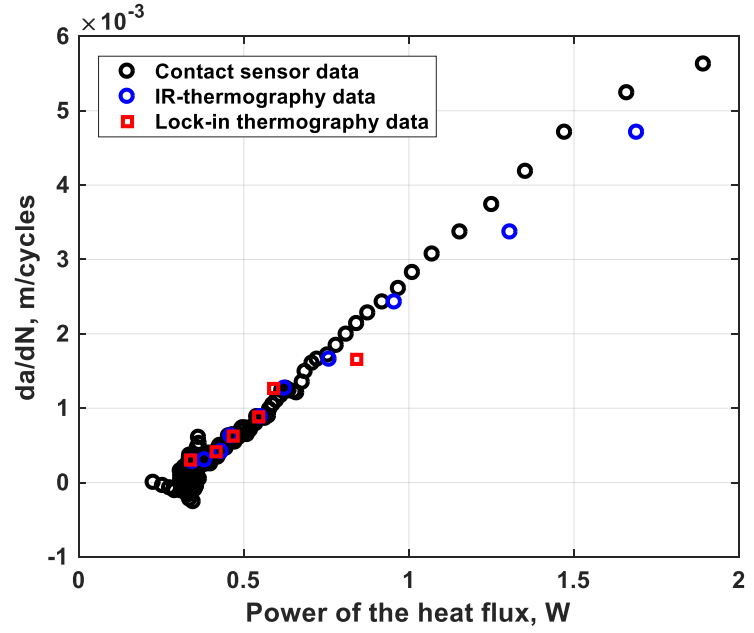

(a)

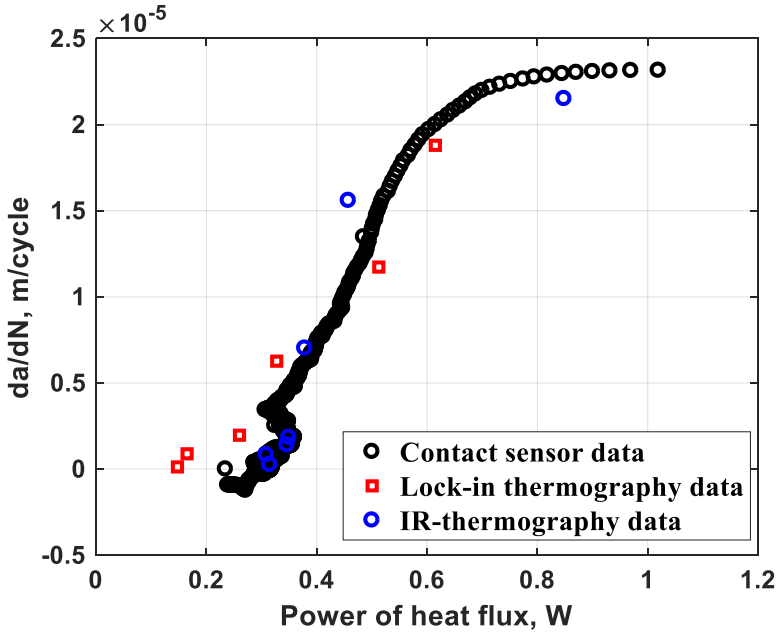

(b)

Fig. 5. Dependency between crack growth rate and power of heat for AISI 304 stainless steel (a) and titanium alloy (b).

It can be seen that the dissipated energy measured by lock-in thermography and the heat flux sensor are in good qualitative agreement over the high heat dissipation period. The averaged D-Amplitude values are rising with loading cycles what caused by processes in front of the crack tip.

The D-amplitude behaviour and, in particular, its increase are similar to that of the crack growth rate. Therefore, the value of the D-mode amplitude can be used to describe crack propagation.

Figure 6 presents this relation for titanium alloy Grade 2 and AISI 304 stainless steel obtained during both uniaxial and biaxial loading.

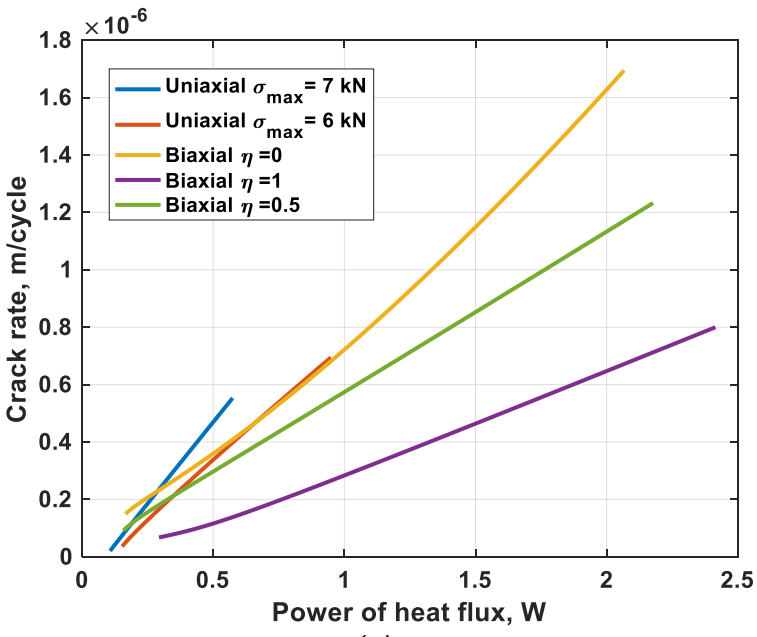

(a)

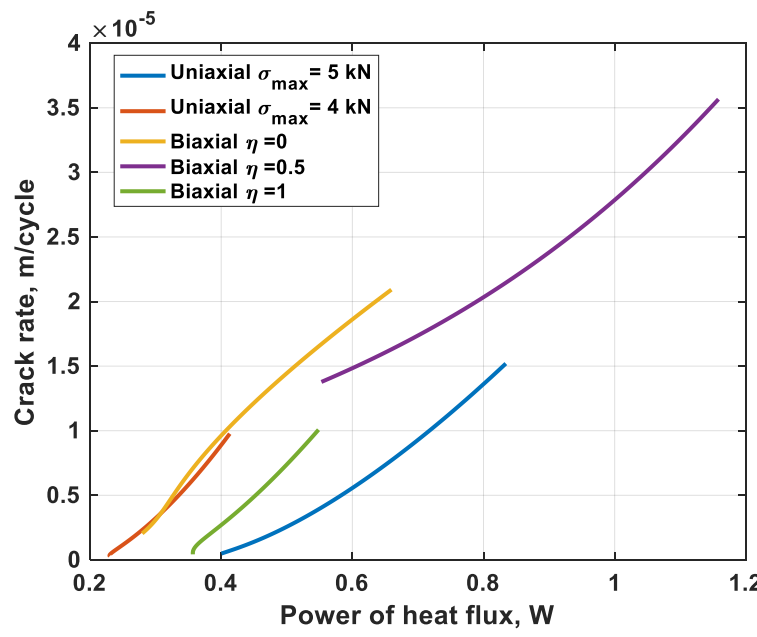

(b)

Fig. 6. Dependency between crack growth rate and power of heat for AISI 304 (a) and titanium alloy Grade 2 (b); $\eta=\sigma_{y} / \sigma_{x}-$ biaxial coefficient.

Analysis of the results has revealed that these techniques can be used for evaluating the crack growth rate under mixed mode loading. 


\section{Conclusion}

IR-thermography data, lock-in thermography data and heat flux sensor measurements were used to investigate energy dissipation under fatigue crack propagation in stainless steel AISI 304 and titanium alloy Grade 2. Comparison of the obtained results demonstrates that they are in good qualitative agreement. The thermography and heat flux sensor measurements showed an increase in the energy dissipated ahead of the crack tip with increasing crack growth rate. The measured values dissipated energy under cyclic loading allows us to determine a linear correlation between the crack rate and dissipated energy (value of amplitude of second temperature harmonics) for predicting the fatigue crack growth under complex loading histories.

\section{REFERENCES}

[1] Iziumova A, Plekhov O. "Calculation of the energy J-integral in plastic zone ahead of a crack tip by infrared scanning". FFEMS. - Vol. 37, pp. 1330-1337, 2014.

[2] Vshivkov A, Iziumova A, Bar U, Plekhov O. "Experimental study of heat dissipation at the crack tip during fatigue crack propagation". Fract Struct Integr. - Vol. 35, pp. 131-137, 2016.

[3] Pradere C, Joanicot M, Batsale J-C, Toutain J, Gourdon C. "Processing of temperature field in chemical microreactors with infrared thermograph". QIRT Journal. - Vol. 3, pp. 117-135, 2006.

[4] Wang C, Blanche A, Wagner D, Chrysochoos A, Bathias C. "Dissipative and microstructural effects associated with fatigue crack initiation on an Armco iron”. Int J Fatigue. - Vol. 58, pp. 152-157, 2014.

[5] Iziumova A, Plekhov O, Vshivkov A, Prokhorov A, Uvarov S. "Studying the Rate of Heat Dissipation at the Vertex of a Fatigue Crack". Tech Phys Lett. - Vol. 40, pp. 810-812, 2014.

[6] lino Y. "Fatigue crack propagation work coefficient - a material constant giving degree of resistance to fatigue crack growth". Eng Fract Mech. - Vol. 12, pp. 279-299, 1979.

[7] Chow C, Lu T. "Cyclic J-integral in relation to fatigue crack initiation and propagation". Eng. Fract. Mech. - Vol. 39, pp. 1-20, 1991

[8] Dowling N, Begley J. "Fatigue Crack Growth During Gross Plasticity and the J-Integral". Mechanics of Crack Growth, ASTM STP 590, American Society for Testing and Materials. pp. 82-103, 1976.

[9] Fedorova A, Bannikov M, Terekhina A, Plekhov O. "Heat dissipation energy under fatigue based on infrared data processing". QIRT J. - Vol. 11, no 1, pp.2-9, 2014.

[10] Plekhov O, Saintier N, Palin-Luc T, Uvarov SV, Naimark OB. "Theoretical analysis, infrared and structural investigations of energy dissipation in metals under cyclic loading”. Mat Sci Eng. - Vol. 462, no 1, pp. 367-369, 2007.

[11] Iziumova A, Vshivkov A, Prokhorov A, Plekhov O, Venkatraman B. "Study of heat source evolution during elastic-plastic deformation of titanium alloy Ti-0.8Al-0.8Mn based on contact and non-contact measurements". PNRPU Mechanics Bulletin. - Vol. 1, pp. 68-81, 2016.

[12] Plekhov O, Vshivkov A, Iziumova A, Zakharov A, Shlyannikov V. "The experimental study of energy dissipation during fatigue crack propagation under biaxial loading”. Fract Struct Integr. - Vol. 48, pp. 50-57, 2019.

[13] Brémond P. "New developments in Thermo Elastic Stress Analysis by Infrared Thermography". IV PanAmerican Conference for Non Destructive Testing, Buenos Aires, 2007.

[14] Sakagami T, Kubo S, Tamura E, Nishimura T. "Identification of plastic-zone based on double frequency lock-in thermographic temperature measurement". In: International conference of fracture ICF 11, 2005.

[15] Bar J. "Determination of dissipated energy in fatigue crack propogetion experiments with Lock-in thermography and heat flow measurements". Proc Structl Int. - Vol. 2, pp. 2105-2112, 2016.

[16] De Finis R, Palumbo D, Ancona F, Galietti U. "Fatigue behaviour of stainless steels: a multi-parametric approach". Proc. of the Society for Experimental Mechanics Series. - Vol. 9, pp. 1-8, 2016.

[17] De Finis R, Palumbo D, Galietti U. "Energetic approach to study the plastic behaviour in CT specimens". Paper QIRT. pp.133, 2018.

[18] Bär J, Seifert S. "Investigation of energy dissipation and plastic zone size during fatigue crack propagation in a high-alloyed steel”. Procedia Mater Sci. - Vol. 3, pp. 408-413, 2014.

[19] Bär J, Seifert S. "Thermographic investigation of fatigue crack propagation in a high-alloyed steel". Advanced Materials Research. - Vol. 892, pp. 936-941, 2014.

[20] Bär J. "Determination of dissipated energy in fatigue crack propagation experiments with lock-in thermography". Procedia Struct Integrity. - Vol. 2, pp. 2105-12, 2016.

[21] Nayeb-Hashemi H, Swet D, Vaziri A. "New electrical potential method for measuring crack growth in nonconductive materials". Measurement. - Vol. 36, no 2, pp. 21-129, 2004.

[22] Boulanger, T., Chrysochoos, A., Mabru, C., Galtier, A., "Calorimetric analysis of dissipative and thermoelastic effects associated with the fatigue behavior of steels". Int. J. Fatigue. - Vol. 26, pp. 221-229, 2004.

[23] Chrysochoos, A., Louche, H., "Infrared image processing to analyze the calorific effects accompanying strain localization”. Int. J. Eng. Sci. - Vol. 38, pp. 1759-1788, 2000.

[24] Fedorova, A.Y., Bannikov, M. V., Terekhina, A.I., Plekhov, O.A. "Heat dissipation energy under fatigue based on infrared data processing". Quant. Infrared Thermogr. J. - Vol. 11, no 1, pp. 2-9, 2014. 